\title{
A Generalization of the Goldman Equation, Including the Effect of Electrogenic Pumps
}

JOHN A. JACQUEZ

Department of Physiology, The Medical School, and Department

of Biostatistics, The School of Public Health, The University

of Michigan, Ann Arbor, Michigan

\begin{abstract}
An equation similar to the Goldman equation is derived for the steady-state diffusion of univalent ions across a membrane with arbitrary potential profile and in the presence of electrogenic pumps. The presence of electrogenic pumps adds a term proportional to the net pump flux to both numerator and denominator in the Goldman equation. For arbitrary potential profiles the permeabilities of the positive ions are all divided by one potential-dependent factor and the permeabilities of the negative ions are divided by another such factor. Both factors are determined by the potential function and tend to vary inversely. If the symmetric part of the potential function is zero, both factors are equal to 1.0. Hence in the general form of the Goldman equation the relative contributions of the positive and negative ions are weighted by factors that are easily calculated if the potential function is known.
\end{abstract}

\section{INTRODUCTION}

Since Goldman [1] published his paper in 1943, the constant field equation has been used widely, although the constant field assumption has been criticized repeatedly as being unrealistic. In his book, Cole [2] comments on the widespread use and the limitations of the constant field equation. Nonetheless, the constant field equation has been used for obtaining relative permeabilities in steady states and in the transient state at the peak of the action potential [3-6], at which point net current flow across the membrane is zero.

Many of the recent criticisms of the constant field equation have been directed to the question of the strict applicability of the constant field assumption. Zelman [7] pointed out a mathematical inconsistency: the strict electroneutrality assumption in conjunction with the Nernst-Planck flux equations leads to an overdetermined set of equations. Friedman [8] showed that the constant field assumption holds rigorously for an uncharged membrane if univalent ions are present only if the total concentration of ions is independent of distance in the membrane and that in 
general this implies that all fluxes are zero. For a charged membrane the constant field equation holds if the uniform charge density in the membrane is sufficient to exclude any coions from the membrane. Recently Arndt et al. [9] have shown that for a neutral membrane and steady state, the constant field assumption is exact for exact electroneutrality if the total number of ions of one valence is the same on both sides of the membrane. Some of these findings have been reported before and may be found in the book by Johnson et al. [10]. In the derivation of the constant field equation it is assumed that the sum of the electrodiffusion fluxes is zero, so that, as has been pointed out by Geduldig [11] and others, it does not hold if electrogenic pumps are present. MacGillivray and Hare [12] develop an asymptotic expansion of the solution for arbitrary potential function in terms of a parameter $\alpha^{-1}$, where $\alpha^{2}=\kappa_{0} k R T / F^{2} N L^{2}, L$ is membrane thickness, $N$ the concentration of fixed charge, $\kappa_{0}$ the permittivity of free space and $\kappa$ the dielectric constant for the membrane. They show that the constant field assumption is a good approximation if $\alpha^{2}$ is large and they conclude that for a $100-\AA$ membrane of lipid the fixed charge concentration must be very low for the assumption to be applicable.

Many of these criticisms miss the mark. For the electrophysiologist the important problem is to define the range of potential profiles for which the constant field equation holds and, when it does not hold, to obtain estimates of the error incurred by using it. The question of constancy of the field is of less importance. In fact, we know that if only univalent ions of one sign are involved, the constant field equation holds for arbitrary potential profile [3, 13, 14] and Barr [14] has shown that if both positive and negative univalent ions are involved, the constant field equation still holds provided the potential profile is an odd function of distance from an origin of coordinates centered in the membrane. In this article I devclop a general solution for the steady-state electrodiffusion of univalent ions across a membrane for arbitrary potential profile and in the presence of electrogenic pumps. From this result it is easy to derive the conditions under which the constant field equation holds and incidentally to examine the effect of nonconstant ionic mobilities.

\section{THEORY}

\section{The Constant Field Equation for the Diffusion Potential}

First I recapitulate the standard derivation $[10,15]$ in brief, to serve as a basis for later argumentation. Let us assume an infinite plane membrane of thickness $a$ separating two solutions. For purposes of symmetry it will be convenient for us to place the origin of coordinates at the center of the membrane, so the membrane extends from $x=-a / 2$ to $x=a / 2$. 
This simplifies some of the derivations but does not affect the final results. Let us start with the Nernst-Planck flux equation for the $k$ th univalent ion:

$$
-J_{k}=u_{k}\left(\frac{R T d c_{k}}{F d x}+z_{k} c_{k} \frac{d \phi}{d x}\right) .
$$

In Eq. (1) $J_{k}$ is the flux of the $k$ th ion, $u_{k}$ its mobility in the membrane, $z_{k}$ its sign, $c_{k}$ its concentration, and $\phi(x)$ is the electrical potential at $x$. The electric field is assumed to vary only in the $x$ direction. Another equation that must hold is Poisson's equation:

$$
\frac{d^{2} \phi}{d x^{2}}=-\frac{\rho(x)}{\kappa \kappa_{0}}
$$

In Eq. (2) $\rho(x)$ is the charge density, $\kappa$ the dielectric constant of the medium, and $\kappa_{0}$ the permittivity of free space. The constant field assumption is equivalent to assuming strict electroneutrality at all points, so that the charge density is zero and Poisson's equation reduces to Laplace's equation. Then, if $V$ is the potential difference across the membrane, Eq. (3) gives the constant field.

$$
\frac{d \phi}{d x}=\frac{V}{a} .
$$

We also assume that only univalent ions have nonzero fluxes and that a steady state holds, so that $J_{k}$ is independent of $x$. Then Eq. (1) becomes a first-order linear differential equation in the concentration and may be integrated to give

$$
\begin{aligned}
c_{k}\left(\frac{a}{-}\right) \exp \left(\frac{z_{k} F V}{2 R T}\right)-c_{k}\left(\frac{-a}{2}\right) & \exp \left(\frac{-z_{k} F V}{2 R T}\right) \\
= & -\frac{a J_{k}}{z_{k} V u_{k}}\left[\exp \left(\frac{F z_{k} V}{2 R T}\right)-\exp \left(\frac{F z_{k} V}{2 R T}\right)\right] .
\end{aligned}
$$

Solving for the flux and factoring out a factor of $\exp \left(-F z_{k} V / 2 R T\right)$ gives

$$
J_{k}=-\left(\frac{z_{k} V u_{k}}{a}\right) \frac{c_{k}(a / 2) \exp \left(z_{k} F V / R T\right)-c_{k}(-a / 2)}{\exp \left(z_{k} F V / R T\right)-1} .
$$

In general, there may be potential jumps between the bulk phases and the adjacent surface of the membrane. The standard assumption $[10,15]$ is that an ion is in equilibrium between bulk and adjacent surface phases. Let the potentials in outer and inner bulk phases be denoted by $\phi_{o}$ and $\phi_{i}$, respectively, and at the membrane surfaces by $\phi(-a / 2)$ and $\phi(a / 2)$. Define the potential jumps as $V_{o}=\phi(-a / 2)-\phi_{o}$ and $V_{i}=\phi_{i}-\phi(a / 2)$. Then the equilibrium assumption gives Eq. (6) for the relations between 
the concentrations in the bulk phases and adjacent surfaces for positive and negative ions, respectively.

$$
\begin{aligned}
c_{k}\left(\frac{a}{2}\right) & =b_{k} c_{i k}(+) \exp \left(\frac{F V_{i}}{R T}\right)=b_{i} c_{i k}(+) Z_{i}, \\
c_{k}\left(-\frac{a}{2}\right) & =b_{k} c_{o k}(+) \exp \left(-\frac{F V_{o}}{R T}\right)=b_{k} c_{o k}(+) Z_{o}^{-1}, \\
c_{j}\left(\frac{a}{2}\right) & =b_{j} c_{i j}(-) \exp \left(-\frac{F V_{i}}{R T}\right)=b_{j} c_{i j}(-) Z_{i}^{-1}, \\
c_{j}\left(-\frac{a}{2}\right) & =b_{j} c_{o j}(-) \exp \left(\frac{F V_{o}}{R T}\right)=b_{j} c_{o j}(-) Z_{o} .
\end{aligned}
$$

In Eq. (6) $c_{i k}(+), c_{o k}(+)$ and $c_{i j}(-)$ and $c_{o j}(-)$ are bulk phase concentrations for $k$ th positive and $j$ th negative ions, respectively, and $b_{k}$ and $b_{j}$ are partition coefficients that are independent of the potential jumps at the surfaces. The permeability is defined by Eq. (7), in which $D_{k}$ is the diffusion coefficient in the membrane for the $k$ th ion.

$$
P_{k}=u_{k} b_{k} R T / a F=D_{k} b_{k} / a \text {. }
$$

The constant field equation is then obtained from the added assumption of zero net current flow across the membrane. If only univalent ions have nonzero fluxes, this is equivalent to the condition, $\Sigma z_{k} J_{k}=0$ and leads to Eq. (8), in which the positive and negative ions are distinguished.

$$
V=\frac{R T}{F} \ln \left[\frac{Z_{o}^{-1} \Sigma P_{k} c_{o k}(+)+Z_{i}^{-1} \Sigma P_{j} c_{i j}(-)}{Z_{i} \Sigma P_{k} c_{i k}(+)+Z_{o} \Sigma P_{j} c_{o j}(-)}\right] \text {. }
$$

The measurable membrane potential is $V_{m}=V_{o}+V+V_{i}$. Substituting $V_{0}=(R T / F) \ln \left[\exp \left(F V_{0} / R T\right)\right.$ and the corresponding exprcssion for $V_{i}$ in the preceding expression and rearranging Eq. (8) leads to Eq. (9).

$$
V_{m}=\frac{R T}{F}\left[\frac{\Sigma P_{k} c_{o k}(+)+Z_{o} Z_{i}^{-1} \Sigma P_{j} c_{i j}(-)}{\Sigma P_{k} c_{i k}(+)+Z_{o} Z_{i}^{-1} \Sigma P_{j} c_{o j}(-)}\right] .
$$

Of course, if either $V_{o}=V_{i}=0$ or if $V_{o}=V_{i}, Z_{o} Z_{i}^{-1}=1$.

The derivations that follow depend on the use of the general form of the first law of the mean for integrals, so we state that law now as a lemma [16].

Lemma (First Law of the Mean for Integrals) Given $f(x)$ and $g(x)$, continuous functions in the closed interval $[a, b]$. Let $f(x)$ have the same sign for all $x$ in $[a, b]$. Then Eq. (10) holds.

$$
\int_{a}^{b} g(x) f(x) d x=g(\zeta) \int_{a}^{b} f(x) d x
$$

Here $\zeta$ is some point in the interval $[a, b]$. Furthermore, $g(\zeta)$ is the mean value of $g(x)$ in $[a, b]$ with respect to $f(x)$ as a weighting function. 


\section{The Effect of Nonconstant Mobilities}

Now let us return to Eq. (1) and assume some arbitrary continuous potential profile. The zero of potential is taken where the straight line connecting $\phi(-a / 2)$ and $\phi(a / 2)$ intersects the midline of the membrane, so that $\phi(a / 2)=-\phi(-a / 2)=V / 2$. Assume that $u_{k}$ may be a function of $x$. Then, integration of the flux Eq. (1) gives Eq. (11) and (12) for positive and negative ions, respectively, instead of Eq. (4).

$$
\begin{aligned}
c_{k}(a / 2) \exp (F V / 2 R T)-c_{k}( & -a / 2) \exp (-F V / 2 R T) \\
& =-\frac{F J_{k}}{R T} \int_{-a / 2}^{a / 2} \frac{\exp \lfloor F \phi(x) / R T\rfloor}{u_{k}} d x, \\
c_{j}(a / 2) \exp (-F V / 2 R T)- & c_{j}(-a / 2) \exp (F V / 2 R T) \\
= & -\frac{F J_{j}}{R T} \int_{-a / 2}^{a / 2} \frac{\exp [-F \phi(x) / R T]}{u_{j}} d x .
\end{aligned}
$$

Since $\exp [F \phi(x) / R T]$ and $\exp [-F \phi(x) / R T]$ are both positive everywhere in $[-a / 2, a / 2]$, the integrals in Eq. (11) and (12) may be written in terms of the mean values of $1 / u_{k}$ and $1 / u_{j}$ as defined by Eqs. (13) and (14).

$$
\begin{gathered}
\int_{-a / 2}^{a / 2} \frac{\exp [F \phi(x) / R T]}{u_{k}(x)} d x=\frac{1}{\tilde{u}_{k}} \int_{-a / 2}^{a / 2} \exp \left[\frac{F \phi(x)}{R T}\right] d x=\frac{\mathrm{I}}{\tilde{u}_{k}}, \\
\int_{-a / 2}^{a / 2} \frac{\exp [-F \phi(x) / R T]}{u_{j}(x)} d x=\frac{1}{\tilde{u}_{j}} \int_{-a / 2}^{a / 2} \exp \left[-\frac{F \phi(x)}{R T}\right] d x=\frac{\mathrm{II}}{\tilde{u}_{j}} .
\end{gathered}
$$

For our purposes $\tilde{u}_{k}$ and $\tilde{u}_{j}$ are not the mean values of $u_{k}$ and $u_{j}$ but are explicitly defined by Eqs. (13) and (14). Note that the mean values $1 / \tilde{u}_{k}$ and $1 / \tilde{u}_{j}$ are with respect to different weighting functions for the positive and for the negative ions. Solving Eqs. (11) and (12) for the fluxes leads then to Eqs. (15) and (16) for the positive and negative ions.

$J_{k}=\left(-a P_{k} / I\right)\left[Z_{i} c_{i k}(+) \exp (F V / 2 R T)-Z_{o}^{-1} c_{o k}(+) \exp (-F V / 2 R T)\right]$,

$J_{j}=\left(-a P_{j} / I I\right)\left[Z_{i}^{-1} c_{i j}(-) \exp (-F V / 2 R T)-Z_{o} c_{o j}(-) \exp (F V / 2 R T)\right]$.

$P_{k}$ and $P_{j}$ are defined as in Eq. (7) except that $\tilde{u}_{k}$ and $\tilde{u}_{j}$ replace $u_{k}$ and $u_{j}$. If the mobilities are constant, the permeabilities $P_{k}$ and $P_{j}$ are constant. If the mobilities are functions of $x$ in the membrane, the permeabilities become functions of the potential profiles because the mean values $1 / \tilde{u}_{i}$ and $1 / \tilde{u}_{j}$ are taken with respect to weighting functions that depend on the potential profiles. Thus it is clcar that no matter what the potential function, if the mobilities are not constant, the permeabilities become functions of the potential profiles. 
The Flux Equations for Arbitrary Potential Profiles

Now consider the flux equations, (15) and (16). Integrals I and II are related in a unique way no matter what the potential profile is. To show this, note that any potential function may be written as a sum of an even (symmetric) and an odd (antisymmetric) function, as in Eq. (17).

$$
\phi(x)=\frac{1}{2}[\phi(x)+\phi(-x)]+\frac{1}{2}[\phi(x)-\phi(-x)] .
$$

The first term of Eq. (17), $\sigma(x)=\frac{1}{2}[\phi(x)+\phi(-x)]$, is an even function of $x, \sigma(x)=\sigma(-x)$, such that $\sigma(-a / 2)=\sigma(a / 2)=0$. The second term in Eq. (17), $\theta(x)-\frac{1}{2}[\phi(x)-\phi(-x)]$, is an odd function, $\theta(x)=-\theta(-x)$. Using the mean value law, integrals I and II may be written as in Eqs. (18) and (19).

$$
\begin{aligned}
& I=\int_{-a / 2}^{a / 2} \exp \left[\frac{F \sigma(x)}{R T}\right] \exp \left[\frac{F \theta(x)}{R T}\right] d x \\
&=g(\xi) \int_{-a / 2}^{a / 2} \exp \left[\frac{F \theta(x)}{R T}\right] d x, \\
& I I=\int_{-a / 2}^{a / 2} \exp \left[-\frac{F \sigma(x)}{R T}\right] \exp \left[-\frac{F \theta(x)}{R T}\right] d x \\
&=h(\zeta) \int_{-a / 2}^{a / 2} \exp \left[-\frac{F \theta(x)}{R T}\right] d x .
\end{aligned}
$$

Here $g(\xi)=\exp [F \sigma(\xi) / R T], h(\zeta)=\exp [-F \sigma(\zeta) / R T]$ where $\xi$ and $\zeta$ fall in the interval $[-a / 2, a / 2]$. If we make the change of variable $y=-x$ in the integral on the right of Eq. (19), it is easy to show the equality given by Eq. (20).

$$
\int_{-a / 2}^{a / 2} \exp \left[\frac{F \theta(x)}{R T}\right] d x=\int_{-a / 2}^{a / 2} \exp \left[-\frac{F \theta(x)}{R T}\right] d x=\mathbf{\Omega} .
$$

Relation (20) is true for all antisymmetric functions $\theta(x)$. Hence of $\phi(x)$ is antisymmetric, $\sigma(x)=0$, and $g=h=1$, so $\mathrm{I}=\mathrm{II}$, a result that has already been obtained by Barr [14].

Furthermore, by making the change of variable $y=-x$ it is easy to show that integral II is also given by Eq. (21).

$$
I I=\int_{-a / 2}^{a / 2} \exp \left[-\frac{F \sigma(x)}{R T}\right] \exp \left[\frac{F \theta(x)}{R T}\right] d x
$$

Thus $g(\xi)=g$ is the mean value of $\exp [F \sigma(x) / R T]$ with respect to $\exp [F 0(x) /$ $R T]$ and $h=h(\zeta)$ is the mean value of the inverse of $\exp [F \sigma(x) / R T]$ with respect to the same function. It is tempting to speculate that $g h=1$. This is not so but, as might be expected, it turns out that $g h$ is approximately 1 for many potential functions and $g$ and $h$ vary inversely as the potential function is changed. Thus $\mathbf{I}=g \Omega, \mathrm{II}=h \Omega$. 


\section{The Generalized Goldman Equation}

Suppose electrogenic pumps are present, so that the steady-state pump flux in equivalents of univalent positive ions per unit area of membranc is $J_{p}$.

$$
0=\sum_{k} J_{k}(+)-\sum_{j} J_{j}(-)+J_{p} .
$$

Note that the fluxes of positive and negative ions are defined to be positive when directed inward from outside to inside. Therefore, $J_{p}$ is the equivalent pump flux of positive ions, positive if inward, negative if outward. Combining Eqs. (15), (16), and (22) gives us Eq. (23).

$$
\begin{aligned}
0= & -\sum_{k}\left(P_{k} / g\right)\left[Z_{i} c_{i k}(+) \exp (F V / 2 R T)-Z_{o}^{-1} c_{o k}(-) \exp (-F V / 2 R T)\right] \\
& +\sum_{j}\left(P_{j} / h\right)\left[Z_{i}^{-1} c_{i j}(-) \exp (-F V / 2 R T)-Z_{o} c_{o j}(-) \exp (F V / 2 R T)\right] \\
& +\left(J_{p} \Omega / a\right) .
\end{aligned}
$$

The integral $\Omega$, which is the integral of $\exp [F \theta(x) / R T]$ across the membrane, may be evaluated as follows. Write the antisymmetric part of the potential function as a sum of the linear portion and a remainder, as in Eq. (24).

$$
\theta(x)=(V x / a)+\omega(x) .
$$

From this definition, $\omega(a / 2)=\omega(-a / 2)=0$ and $\omega(0)=0$ because $\omega(x)$ is antisymmetric. Applying the mean value theorem gives Eq. (25).

$$
\begin{aligned}
& \Omega=\int_{-a / 2}^{a / 2} \exp \left(\frac{F \omega(x)}{R T}\right) \exp \left(\frac{F V x}{a R T}\right) d x=w \int_{-a / 2}^{a / 2} \exp \left[\frac{F V x}{R T}\right] d x, \\
& \Omega=\frac{w a R T}{F V}\left[\exp \left(\frac{F V}{2 R T}\right)-\exp \left(-\frac{F V}{2 R T}\right)\right] .
\end{aligned}
$$

If the antisymmetric part of the potential is linear, $\omega(x)=0$ and $w=1$. Substituting for $\Omega$ in Eq. (23) and rearranging terms gives the general equivalent of the Goldman equation for arbitrary potential profile and for electrogenic pump flux $J_{p}$.

$$
V=\frac{R T}{F} \ln \left[\frac{Z_{o}^{-1} \Sigma_{k}\left(P_{k} / g\right) c_{o k}(+)+Z_{i}^{-1} \Sigma_{j}\left(P_{j} / h\right) c_{i j}(-)-\left(J_{p} R T w / F V\right)}{Z_{i} \Sigma_{h}\left(P_{k} / g\right) c_{i k}(+)+Z_{o} \Sigma_{j}\left(P_{j} / h\right) c_{o j}(-)-\left(J_{p} R T w / F V\right)}\right] .
$$

By the sign convention for current, a current (of positive charge) is positive when directed out of the membrane, whereas the pump flux is defined to be positive for a net flow of positive ions into a cell; so if $i_{p}$ is the net pump current density, $i_{p}=-F J_{p}$ and all terms in the numerator and denominator of Eq. (26) have a positive sign, as in Eq. (27). At first sight Eq. (26) appears to be of little help if an electrogenic pump is present because $V$ 
appears on both sides of the equation. But $J_{p}$ is often measurable and $V$ may be approximated by the membrane potential. Furthermore, $i_{p} / V=$ $-F J_{p} / V$ is the equivalent conductance of the transport process working across a potential difference $V$, so that Eq. (26) takes into account the potential dependence of the active transport process. Thus Eq. (26) retains the form of the Goldman equation and explicitly exhibits the effects of the nonlinear portions of the potential function in the factors $g, h$, and $w$ as well as the effects of any electrogenic pumps, including the potential dependence of the net pump conductance.

\section{The Membrane Potential}

The potential $V$ in Eq. (26) is the potential across the membrane, $V=\phi(a / 2)-\phi(-a / 2)$. To obtain the measurable membrane potential, add $V_{o}$ and $V_{i}$, as was done in obtaining Eq. (9), to obtain Eq. (27), in which the pump current density has been substituted for $-J_{p} F$.

$V_{m}=\frac{R T}{F} \ln \left[\frac{\Sigma_{h}\left(P_{k} / g\right) c_{o k}(+)+Z_{o} Z_{i}^{-1} \Sigma_{j}\left(P_{j} / h\right) c_{i j}(-)+\left(i_{p} R T w Z_{0} / V F^{2}\right)}{\Sigma_{k}\left(P_{k} / g\right) c_{i k}(+)+Z_{o} Z_{i}^{-1} \Sigma_{j}\left(P_{j} / h\right) c_{o j}(-)+\left(i_{p} R T w Z_{i}^{-1} / V F^{2}\right)}\right]$.

Thus if $i_{p}=0$, an equation similar to the Goldman equation holds for arbitrary potential profile with apparent permcabilities $p_{k}-P_{k} / g$ for univalent positive ions and $p_{j}=Z_{o} Z_{i}^{-1} P_{j} / h$ for univalent negative ions. Two interesting and perhaps useful general conclusions follow. Ratios of apparent permeabilities of ions of like sign are ratios of true permeabilities, for if $k$ and $m$ refer to ions of the same sign, $p_{k} / p_{m}=P_{k} / P_{m}$. If the potential jumps at the interfaces differ little, $Z_{o} Z_{i}^{-1} \simeq 1$, then the products of apparent permeabilities of ions of opposite sign will be approximately equal to products of true permeabilities for a wide range of potential functions for which $g h \simeq 1$. Incidentally, if $V_{o}-V_{i}=1 \mathrm{mV}, Z_{o} Z_{i}^{-1}=1.04$ at $37^{\circ} \mathrm{C}$ and if $V_{o}-V_{i}=5 \mathrm{mV}, Z_{o} Z_{i}^{-1}=1.2$. For $J_{p}=0$, Patlak [17] has derived an equation similar to Eq. (27) but his apparent permeabilities are rate coefficients, which include a nonspecified dependence on the potential and the concentrations.

In the derivations I have used concentrations throughout. The equations are rigorously true if concentrations are replaced by activities.

\section{DISCUSSION AND CONCLUSIONS}

Now let us examine and summarize the nature of the solution, Eq. (27), for different types of membranes, and characterize the solution in terms of the values that $g, h$, and $w$ assume. In order to obtain some feel for the variation of $g, h$, and $w$ with changes in the potential function, a number 
of potential functions were chosen arbitrarily. However, one of the current pictures of the cell membrane is included, that which views the cell membrane as a neutral lipid membrane with a dipole layer due to the charged groups on the phospholipids on each surface, the two dipole layers being oppositely oriented. In the computations that are cited I assume a $100 \AA$-thick membrane of dielectric constant 5 and $V=100 \mathrm{mV}$. The mKS system of units was used throughout.

\section{Overall Neutral Membranes}

\section{Microscopic neutrality}

If the membrane is everywhere neutral, the charge density $\rho(x)$ is zero everywhere in the membrane. Then the potential is the constant field potential, $\phi=V x / a, g=h=1$, and $w=1$. If $J_{p}=0$, the constant field equation holds with apparent permeabilities $p_{k}=P_{k}$ and $p_{j}=Z_{o} Z_{i}^{-1} P_{j}$ for positive and negative ions, respectively.

\section{Macroscopic neutrality}

Assume the charge density is not zero everywhere but the net charge is zero:

$$
\int_{-a / 2}^{a / 2} \rho(x) d x=0
$$

Then the potential is not linear. There are two cases to consider.

1. Antisymmetric charge distribution. If $\rho(x)$ is antisymmetric, then from Poisson's equation it follows that the second derivative of the potential $\phi^{\prime \prime}(x)$ is antisymmetric. Integrating twice, we see that the symmetric part of $\phi(x)$ must be a constant. But $\sigma(a / 2)=0$; hence, $\sigma(x)=0$ and $\phi(x)$ must be antisymmetric. Therefore $g=h=1$ and if $J_{p}=0$, the constant field equation holds. If $J_{p} \neq 0$, the size of the term in $J_{p}$ depends on the value of $w$ as well as on $J_{p}$. Note that $w$ is determined by the antisymmetric part of the potential only; it is independent of the symmetric part. To obtain some idea of values $w$ can attain I have calculated $w$ for

$$
\omega(x)=0 \cdot \ln \left[(2 x / a)^{3}-(2 x / a)\right] \quad \text { and } \quad V=-0.1 \mathrm{~V} .
$$

The potential $\omega(x)$ is given by a linear charge density, $\rho(x)=-212.5 .10^{12}$ $n x \mathrm{C} / \mathrm{m}^{3}$. For $n=-1$ this corresponds to a positive charge in the right half of the membrane of 1.66 positrons per $100 \AA \times 100 \AA$ of surface, the charge density increasing linearly from 0 at $x=0$ to $106.25 \mathrm{C} / \mathrm{m}^{2}$ at $x=50 \AA$; the left half of the membrane has the same negative charge density. For this case $w=0.713$. If the negative charge is placed on the right half of the membrane and the positive on the left half, $w$ becomes 2.48. To take an example that is closer to a standard picture of the cell 
membrane I chose a distributed charge density equivalent to a diffuse dipole layer at each surface given by Eq. (29), in which $a=10^{-8} \mathrm{~m}(100 \AA)$. $\rho(x)=$

$$
\left\{\begin{array}{lc}
0.265 .10^{3} n\left(\frac{x}{a}-0.4\right)\left(\frac{x}{a}-0.45\right)\left(\frac{x}{a}-0.5\right), & 0.4 a \leqslant x \leqslant 0.5 a, \\
0, & -0.4 a<x<0.4 a, \\
0.265 .10^{3} n\left(\frac{x}{a}+0.4\right)\left(\frac{x}{a}+0.45\right)\left(\frac{x}{a}+0.5\right), & -0.5 a \leqslant x \leqslant-0.4 a .
\end{array}\right.
$$

For $n=400$ this corresponds to a dipole of 1 electron and 1 positron per 25 square angstroms on each side of the membrane, distributed as given by $\rho(x)$. Note that for the right half of the membrane the negative charge is distributed between $0.4 a$ and $0.45 a$ and the positive charge between $0.45 a$ and $0.5 a$, the centers of the two charge distributions being $5 \AA$ apart. This corresponds to a dipole moment density of $32 \cdot 10^{-11}$ coulomb-meter per square meter on each side of the membrane. For $n \leqslant 400$ with $V=$ $-0.1 \mathrm{~V}, 0.995<w<1.005$.

2. Charge distribution not antisymmetric. Then the even part of $\phi^{\prime \prime}$ is not zero, but from Eq. (28) it follows that $\int_{0}^{a / 2} \sigma^{\prime \prime} d x=0$ and $\sigma$ changes sign at least once in each of the intervals $[-a / 2,0],[0, a / 2]$. This also implies that $\sigma^{\prime}(-a / 2)=\sigma^{\prime}(a / 2)=0$. If $\sigma^{\prime \prime}(x)$ has many sign changes in $[-a / 2, a / 2]$, since $\sigma$ has two less sign changes than $\sigma^{\prime \prime}$, there are many such potential profiles for which both $g$ and $h$ are close to 1.0. To show the effect of sign changes in $\sigma(x)$, I have calculated $g$ and $h$ for $\theta(x)=$ $0 . \ln \left[(2 x / a)^{3}-(2 x / a)\right]-0.1(x / a)$ and the symmetric part of the potential given by Eq. (30).

$$
\begin{aligned}
& \sigma_{1}(x)=-2.155 \mathrm{~m}[\cos (2 \pi x / a)+1], \\
& \sigma_{2}(x)=1.078 \mathrm{~m}[\cos (4 \pi x / a)-1], \\
& \sigma_{3}(x)=0.539 \mathrm{~m}[\cos (8 \pi x / a)-1], \\
& \sigma_{4}(x)=0.2694 \mathrm{~m}[\cos (16 \pi x / a)-1],
\end{aligned}
$$

For $m=1$ each consists of a total charge of 1 electron and 1 positron per $100 \AA \times 100 \AA$ of membrane surface distributed according to a charge density $\rho(x)=\kappa \kappa_{o} \sigma^{\prime \prime}(x)$. The values of $g$ and $h$ obtained for $n=0,1,-1$, all for $m=1$, are given in Table 1. Note that as the number of sign changes in $\sigma(x)$ increases, $g$ and $h$ come closer together and in fact both approach the value 1.0; and that this is true when the antisymmetric part of the potential function is linear $(n=0)$ and when it contains substantial nonlinear components $(n=+1,-1)$. 
TABLE I

VALUES OF $g$ AND $h$ FOR THE SYMMETRIC POTENTIAL FUNCTIONS GIVEN IN EQ. (30) AND ANTISYMMETRIC POTENTIAL $\theta^{a}$ FOR A NEUTRAL MEMBRANE

\begin{tabular}{|c|c|c|c|c|c|c|}
\hline \multirow{2}{*}{$\begin{array}{c}\text { Symmetric } \\
\text { potential } \\
\text { function }\end{array}$} & \multicolumn{2}{|c|}{$n=0$} & \multicolumn{2}{|c|}{$n=1$} & \multicolumn{2}{|c|}{$n=-1$} \\
\hline & $g$ & $h$ & $g$ & $\bar{h}$ & $g$ & $h$ \\
\hline$\sigma_{1}$ & 0.425 & 14.69 & 0.372 & 19.21 & 0.385 & 10.63 \\
\hline$\sigma_{2}$ & 0.484 & 3.58 & 0.376 & 4.39 & 0.498 & 3.58 \\
\hline$\sigma_{3}$ & 0.634 & 1.82 & 0.612 & 1.88 & 0.656 & 1.76 \\
\hline$\sigma_{4}$ & 0.779 & 1.33 & 0.776 & 1.34 & 0.784 & 1.32 \\
\hline
\end{tabular}

${ }^{a} \theta=0.1 n\left[(2 x / a)^{3}-(2 x / a)\right]-0.1 x / a$.

\section{Membranes with Net Charge}

Let $\bar{\rho}$ be the mean net charge density. Then the charge density may be written as $\bar{\rho}+\rho(x)$ where $\rho(x)$ has zero mean value across the membrane. Thus Poisson's equation may be written as in Eq. (31).

$$
\frac{d^{2} \phi}{d x^{2}}=-\frac{(\bar{\rho}+\rho(x))}{\kappa \kappa_{a}}=C+\sigma^{\prime \prime}(x)+\theta^{\prime \prime}(x)
$$

where $\sigma^{\prime \prime}(x)$ and $\theta^{\prime \prime}(x)$ have the properties of those of an overall neutral membrane; $C+\sigma^{\prime \prime}(x)$ is the symmetric part of $\phi^{\prime \prime}(x)$. Then the potential function may be written as in Eq. (32).

$$
\phi(x)=-\frac{C}{2}\left(\frac{a^{2}}{4}-x^{2}\right)+\sigma(x)+\theta(x) .
$$

The first term in Eq. (32) is the contribution from the uniform net charge density $\bar{\rho}$ and $\sigma(a / 2)=\sigma(-a / 2)=0, \theta(a / 2)=V / 2, \theta(-a / 2)=-V / 2$. Integral $I$ is then given by Eq. (33).

$$
I=\int_{-a / 2}^{a / 2} \exp \left\{\left[-\frac{C\left(a^{2} / 4-x^{2}\right)}{2+\sigma(x)}\right] \frac{F}{R T}\right\} \exp \left[\frac{F \theta(x)}{R T}\right] d x .
$$

If the net charge is negative, $C$ is positive, so that the larger $C$ is, the smaller must be the mean value $g(\xi)$ and the larger $h(\zeta)$ must be. But if $C$ is large enough, the terms in the negative ions in Eq. (27) become negligible and if $J_{p}=0$, the constant field equation again holds, no matter what $\sigma(x)$ and $\theta(x)$ are. This result holds because coions are then excluded from the membrane and the fluxes of coions (Eq. (16)) become negligible. The converse is true if the net charge is positive. To obtain some idea of the magnitude of this effect, I have calculated $g$ and $h$ for the linear antisymmetric part of the potential function $\theta(x)=-0.1(x / a)$ and for a uniform positive charge density equivalent to $n$ positrons per $100 \AA \times$ $100 \AA$ of membrane surface but spread uniformly across the membrane. 
Denote the $g$ and $h$ obtained for a charge equal to that of $n$ positrons by $g_{n}$ and $h_{n}$. Then $g_{\frac{1}{2}}=1.649, h_{\frac{1}{2}}=0.651 ; g_{1}=2.904, h_{1}=0.454 ; g_{2}=$ $10.542, h_{2}=0.264 ; g_{5}=945, h_{5}=0.111$. For the same uniform negative charge density $g$ and $h$ should be interchanged. Note that as the uniform positive charge density is increased, the terms for the positive ions in Eq. (27) very soon become negligible in comparison with the terms for the negative ions.

Finally I emphasize that Eq. (27) includes the effects of any electrogenic pumps. Even if the $\mathrm{Na}^{+}-\mathrm{K}^{+}$pump of most cells is not electrogenic, it is possible that the transport systems for some solutes, such as amino acids and sugars, which involve a cotransport of sodium, may be electrogenic in some cells [18-21]. This question has not really been settled yet. If so, Eq. (27) is the generalization of the Goldman equation needed to deal with steady states in which transport of such solutes occurs.

\section{ACKNOWLEDGMENTS}

The author thanks Dr. James Ranck for his critical comments and Mr. Eugene Daniels for programming help. This work was supported in part by grants GM0892 and CA06734 from the National Institutes of Health.

\section{REFERENCES}

1 D. E. Goldman, J. Gen. Physiol. 27(1943), 37.

2 K. S. Cole, Membranes, ions and impulses, Univ. of California Press, Berkeley, 1968.

3 A. L. Hodgkin and P. Horowicz, J. Physiol (London) 148(1959), 127.

4 P. F. Baker, A. L. Hodgkin and T. I. Shaw, J. Physiol. (London) 164(1962), 355.

5 D. E. Chiarandini and E. Stefani, J. Gen. Physiol. 50(1967), 1183.

6 F. A. Dodge and B. Frankenhaeuser, J. Physiol. (London) 148(1959), 188.

7 P. A. Zelman, J. Theoret. Biol. 18(1968), 396.

8 M. H. Friedman, J. Theoret. Biol. 25(1969), 502.

9 R. A. Arndt, J. D. Bond and L. D. Roper, Biophys. J. 10(1970), 1149.

10 F. H. Johnson, H. Eyring and M. I. Polissar, The kinetic basis of molecular biology, Wiley, New York, 1954.

11 D. Geduldig, J. Theoret. Biol. 19(1968), 67.

12 A. D. MacGillivray and D. Hare, J. Theoret. Biol. 25(1969), 113.

13 L. J. Mullins and K. Noda, J. Gen. Physiol. 47(1963), 117.

14 L. Barr, J. Theoret. Biol. 9(1965), 351.

15 R. Plonsey, Bioelectric phenomena, McGraw-Hill, New York, 1969.

16 E. Goursat, $A$ course in mathematical analysis. Dover, New York, 1959.

17 C. Patlak, Nature 188(1960), 944.

18 Gilles-Baillien and E. Schoffeniels, Arch. Intern. Physiol. Biochim. 73(1965), 355.

19 J. F. Quay and W. McD. Armstrong, Proc. Soc. Exptl. Biol. Med. 131(1969), 46.

20 T. Hoshi and Y. Komatsu, Tohuku J. Med. 100(1970), 47.

21 R. C. Rose and S. G. Schultz, Biochim. Biophys. Acta 211(1970), 376. 